\title{
A New Machine Learning Approach to Deblurring License Plate Using K-Means Clustering Method
}

\author{
Sanaz Aliyan ${ }^{1}$ \\ Islamic Azad University, South Tehran Branch, Tehran, Iran
}

\author{
Ali Broumandnia ${ }^{* 2}$ \\ Islamic Azad University, South Tehran Branch, Tehran, Iran
}

\begin{abstract}
Vehicle license plate recognition (LPR) is one of the important fields in Intelligent Transportation Systems (ITS). LPR systems aim to locate, segment and recognize the license plate from captured car image. Despite the great progress of LPR system in the last decade, there are still many problems to solve to reach a robust LPR system adapted to different environment and condition. The current license plate recognition systems will not effectively work well for blurred plate image. In this paper, to overcome the blurring problem a new machine learning approach to Deblurring License Plate using the K-Means clustering method have proposed. Experimental results demonstrate the effectiveness of the K-Means clustering as a feature selection method for license plate images.
\end{abstract}

Keywords- license plate recognition; K-Means clustering; deblurring; machine learning.

\section{INTRODUCTION}

Recently, the vehicle license plate recognition (LPR) technology has developed and applied in our life and has been used extensively in highway and bridge charge, port, airport gate monitoring, and so on. There are many applications for Intelligent Transportation Systems (ITS) such as traffic analysis, parking automation, identification of stolen vehicles, the license plate recognition and, etc. A complete license plate recognition system consists of three major parts: license plate location, license plate segmentation and character recognition [1-3]. The first step locates LP regions and extracts an image containing a plate. The segmentation step separates the symbols or characters from each other in one LP, and the recognition step finally converts the grey-level image block into characters or symbols by pre-defined recognition models. Recognition step refers to image analysis and pattern recognition [4]. Despite the great progress of LPR system in the last decade, there are still many problems to solve to reach a robust LPR system adapted to different environment and condition. Assuming that license plate region is detected in very low resolution (LR), the current license plate recognition systems will not effectively work well [5]. Motion blurring is one of the prime causes of poor image quality in digital imaging. If objects in a scene are moving fast or the camera is moving over the period of exposure time, the objects or the whole scene will look blurry along the direction of relative motion between the object or scene and the camera. Since high resolution (HR) digital cameras are expensive, finding a way to increase the current resolution level is needed. One promising approach is to use signal processing techniques to obtain an HR image (or sequence) from observed multiple low-resolution (LR) images. Recently, such a resolution enhancement approach has been one of the most active research areas, and it is called super resolution (SR) (or HR) image reconstruction. The major advantage of the signalprocessing approach is that it may cost less, and the existing LR imaging systems can be still utilized.

In the past, multi-image based approaches have been proposed to solve motion de-blurring, which recovers clear images from motion-blurred images [5]. In multi-image based SR approaches used multiple low-resolution images is assumed same size images while in LPR case, there are different sizes of LR images because a license plate is usually captured when the vehicle is moving. Hence, images captured at different time instances may provide different perspectives because they were captured at different angles in the field of view. This makes the registration task even more difficult. To overcome this problem we work on single image of license plate using a new machine learning approach using K-Means clustering. In general, a machine learning technique using training data set containing low resolution images predicts a high-resolution image corresponding to its low resolution image. Since Noise and redundancy in the feature space increase the likelihood of overfitting, K-Means clustering is applied to decrease them as a feature selection method.

\section{RELATED WORK}

The first article that clearly introduced the idea of combing SR and LPR to identify moving vehicles was written by Suresh and Kumar [8]. They proposed a little robust super-resolution algorithm is proposed, in which the HR image is modeled as MRF with a discontinuity adaptive regularization (denoted as "DAMRF"). In [9], an alternative generalized model of DAMRF based on the bilateral filtering which connects the bilateral filtering with the Bayesian MAP is proposed. This DAMRF model has edge-preserving and robust to noise from the bilateral filtering. Furthermore, they applied a method for estimating the regularization parameter automatically to the generalized DAMRF super-resolution reconstruction method. Since the prior was non-convex, they used graduated nonconvex (GNC) which is a determining annealing algorithm for performing optimization. However, complex and difficult computational functions of their Maximum a posterior (MAP) based method, prevent it from real-time processing. Later, a fast MAP-based SR algorithm was proposed by Yuan [10]. In this work by using Wiener filter, the computation complexity was decreased to make SR reconstruction, as a de-convolution improvement. But because of removing the priors and 
registration error, this method became unstable. Later, SR based on non-uniform interpolation of registered LR images was proposed by Gambotto [11].The advantage of this work was the fastness and the disadvantage of that was more sensibility to motion estimates. In [12], a unifying approach of SR license plate localization and for LPR to enhance the robust MAP based SR reconstruction excluding mis-registered images is proposed. The quality of registration before including an image to automatic rejection of mis-registered images is verified before the reconstruction step. A learning-based framework has been proposed in [16] for zooming the digits in a license plate, which have been blurred using an unknown kernel. In this paper, the image as an undirected graphical model over image patches is considered. First, they learn the compatibility functions by nonparametric kernel density estimation, using random samples from the training data. Next, the inference problem is solved by using an extended version of the nonparametric belief propagation algorithm. Finally, the super-resolved and restored images are recognized.

In multi-image based SR approaches used multiple lowresolution images is assumed same size images while in LPR case, there are different sizes of LR images because a license plate is usually captured when the vehicle is moving. Hence, images captured at different time instances may provide different perspectives because they were captured at different angles in the field of view. This makes the registration task even more difficult. Another drawback of previous LPR deblurring researches is that the three parts of license plate recognition are not considered, and the plate is cropped manually.

\section{THE PROPOSED APPROACH}

Learning-based techniques attempt to capture the cooccurrence prior between low-resolution and high-resolution image patches. Recently, some studies represented image patches as a sparse linear combination of elements from an over-complete image patch dictionary [13-15]. In [15] they rely on patches from the input image and learn a compact representation for these patch pairs to capture the cooccurrence prior. Their algorithm tries to infer the highresolution image patch for each low-resolution image patch from the input. For this local model, they have two dictionaries Dh and Dl, which are trained to have the same sparse representations for each high-resolution and low-resolution image patch pair.

This method is applied for generic and face images. The experimental results have shown that it is very effective for image patches. Sparse representation is not a suitable algorithm for real time system such as license plate recognition that aims to respond fast, since it solves an optimizing problem to generate a high-resolution image that it is time consuming. In this paper to develop a real time deblurring algorithm based on machine learning approaches is used $\mathrm{K}$ Means clustering. In general, machine learning approaches work based on training data set, thus in a de-blurring license plate system, low-resolution plate images and corresponding high-resolution are considered as training data set. The important step to apply machine learning techniques is defining proper features that in this paper $3 * 3$ patches are used as features. In fact, a de-blurring trained system reconstructs $3 * 3$ patches of low-resolution image one by one to reach a highresolution image. The performance of the system is related on the amount of patches as training data set; however, system that uses large data set is not fast because of redundancy in the feature space. Since there are many same and similar patches in data set, it can be grouped them into some cluster and define the median of the cluster as index patch. Proposed approach consists of two phases. First, training tow impacted dictionary simultaneously from data set using K-Means clustering and then de-blurring input low-resolution license plate image.

\section{A. Joint Dictionary Training}

Given the sampled training image patch pairs, $D S=\{X, Y\}$ where $X=\{x 1, x 2, \ldots, x n\}$ are the sets of sampled highresolution image patches and are $\mathrm{Y}=\{\mathrm{y} 1, \mathrm{y} 2, \ldots, \mathrm{yn}\}$ the corresponding low-resolution image patches (or features), our goal is to learn dictionaries DH and DL for high-resolution and low-resolution image patches In first place, $\mathrm{X}$ is clustered into $\mathrm{K}$ clusters using K-means and then cluster $\mathrm{Y}$ based on their corresponding low-resolution patches. Finally, by the median operator calculate median for each cluster. Figure 1 shows the framework of joint dictionary training.

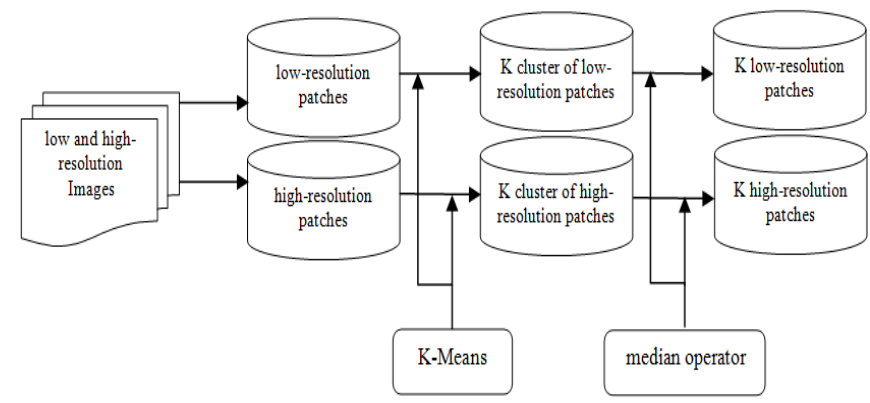

Figure 1. Joint dictionary training framework

\section{B. De-Blurring License Plate Image}

In this phase, low-resolution image is divided into $3 * 3$ patches. De-blurring system find the most similar patch in DL for each patch and then use the corresponding patch in $\mathrm{DH}$ to construct the high-resolution image. The framework of deblurring is shown in figure 2 .

\section{EXPERIMENTAL RESULTS}

In this paper, 151 car images are used as data set as shown in figure 3. A plate location technique is used to extract plate images. This technique work based on edge detection and histogram analysis, as is demonstrated in figure 3 the amount of histograms around the plate is more other parts.

Based on this hypothesize location of plate is detected and extracted. Blurred image is created using a motion filter (Figure 4). These image pairs show our training and testing dataset that is considered 140 and 11 image pairs respectively. The two dictionaries for high-resolution and low-resolution image patches are trained from 272000 patch pairs sampled from training and 140 testing dataset images and fix the dictionaries size to be 2000. Proposed approach is implemented using MATLAB software. 


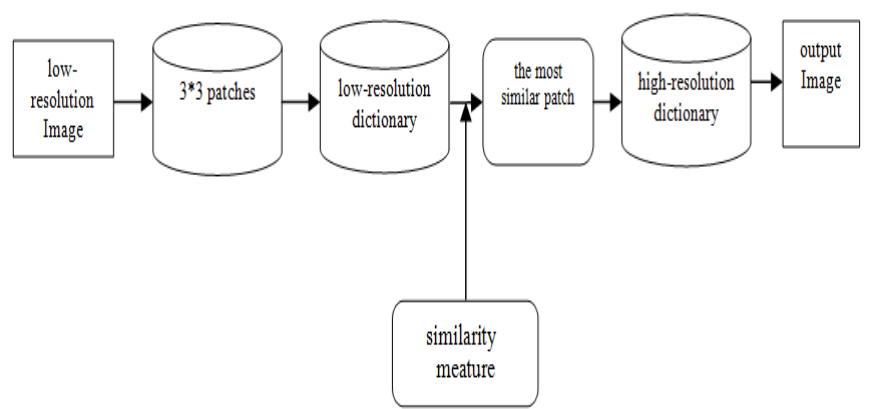

Figure 2. Deblurring License Plate Image Framework
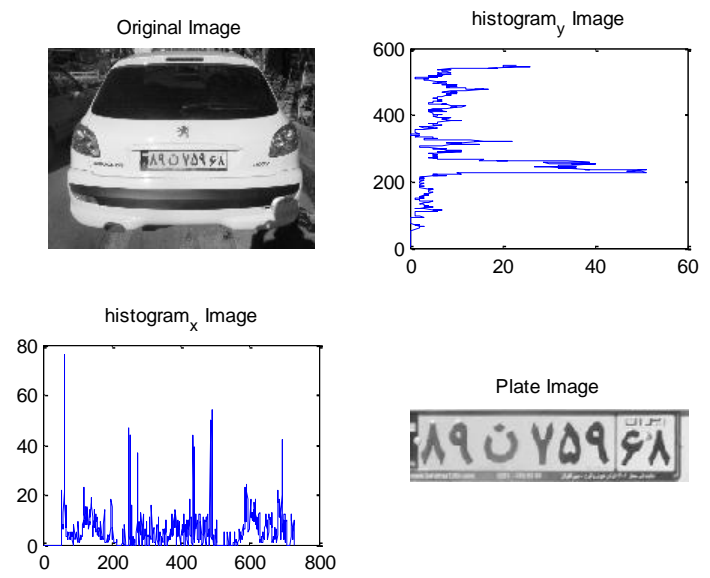

Figure 3. Extracted license plate using plate location technique
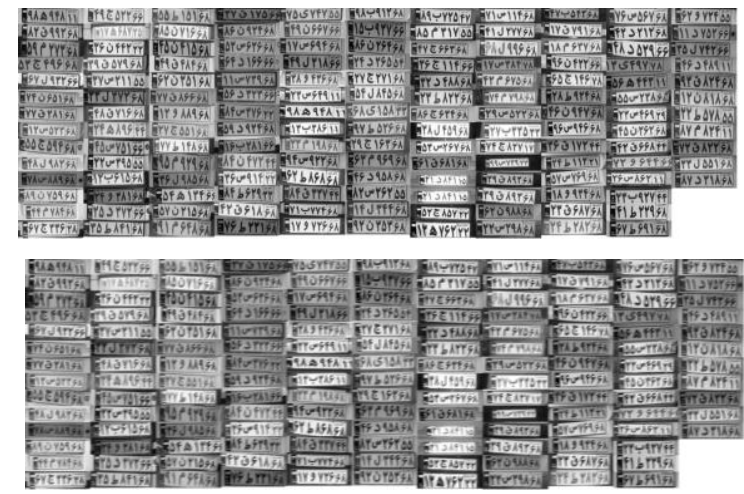

Figure 4. High and low-resolution license plate images

Proposed approach is implemented using MATLAB software.

Figure 5 demonstrates the experimental results for two iterations of applying deblurring algorithm.

\begin{tabular}{|c|c|c|}
\hline Blurred Image & irst Iteration Image & second Iteration Image \\
\hline 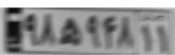 & 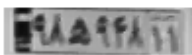 & 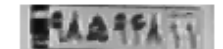 \\
\hline Blurred Image & $\begin{array}{l}\text { (a) } \\
\text { first Iteration Image }\end{array}$ & second Iteration Im age \\
\hline Eiris & ․ㅏㅇㅕ & \\
\hline
\end{tabular}

(b)

\begin{tabular}{|c|c|c|}
\hline Blurred Image & irst Iteration Image & second Iteration Image \\
\hline $8 \%$ C $949 \lambda$ & Aref4G & 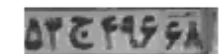 \\
\hline
\end{tabular}

(c)

Figure 5. Deblurring LP images using proposed approach

\section{CONCLUSION}

Associations of super resolution techniques to the current license plate recognition systems have been reported. In this paper a novel approach toward single image SR based upon machine learning technique in terms of coupled dictionaries jointly trained from high- and low-resolution image patch pairs is presented. The compatibilities among adjacent patches are enforced both locally and globally. Experimental results demonstrate the effectiveness of the K-Means clustering as a feature selection method for license plate images. However, one of the most important questions for future investigation is to determine the optimal dictionary size for natural image patches in terms of SR tasks. Tighter connections to the theory of compressed sensing may yield conditions on the appropriate patch size, features to utilize and also approaches for training the coupled dictionaries.

\section{REFERENCES}

[1] F. Wang, L. C. Man, B. Wang, Y. Xiao, W. Pan, and X. Lu, "Fuzzybased algorithm for color recognition of license plates," Pattern Recognit. Lett., vol. 29, no. 7, pp. 1007-1020, May 2008.

[2] V. Abolghasemi and A. Ahmadyfard, "An edge-based color-aided method for license plate detection," Image Vis. Comput., vol. 27, no. 8, pp. 1134-1142, Jul. 2009.

[3] H. Caner, H. S. Gecim, and A. Z. Alkar, "Efficient embedded neuralnetwork-based license plate recognition system," IEEE Trans. Veh. Technol., vol. 57, no. 5, pp. 2675-2683, Sep. 2008.

[4] A. Broumandnia, J. Shanbehzadeh, Fast Zernike wavelet moments for Farsi character recognition, Image and Vision Computing 25 (2007) 717-726.

[5] C. N. E. Anagnostopoulos, I. E. Anagnostopoulos, I. D. Psoroulas, V. Loumos, and E. Kayafas, "License plate recognition from still images and video sequences : A Survey," IEEE Tran. Intell. Transp. Syatem, vol. 9, No. 3, 2008, pp. 377-391.

[6] B. Zitov'a and J. Flusser, "Image registration methods: a survey," Image and Vision Computing, vol. 21, no. 11, pp. 977-1000, 2003.

[7] J. Tian and K.-K. Ma, "A survey on super-resolution imaging," Signal, Image and Video Processing, 2011.

[8] K.V. Suresh, G.M. Kumar, and A.N. Rajagopalan, "Superresolution of License Plates in Real Traffic Videos", IEEE Trans. Intelligent transportation systems, June 2007, Vol. 8, No. 2.

[9] Z. Weili and L. Xiaobo, "A Generalized DAMRF Image Model for Super-Resolution of License Plates", in Photonics and Optoelectronic (SOPO), 2010 Symposium, pp. 1-4.

[10] J. Yuan, S.-D. Du, and X. Zhu, "Fast super-resolution for license plate image reconstruction," in ICPR, IAPR, 2008, pp. 1-4.

[11] J.-P. Gambotto, M. Denis, and R. Livier, "Super-resolution of moving vehicles using an affine motion model," in AVSS, IEEE, 2009, pp. 535540.

[12] N. Chu Duc, M. Ardabilian and C. Liming, "Unifying Approach for Fast License Plate Localization and Super-Resolution", in Pattern Recognition (ICPR), 2010 20th International Conference, pp. 376-379.

[13] Wang, J., Zhu, S., Gong, Y.: Resolution enhancement based on learning the sparse association of image patches. Pattern Recognit.Lett. 31, 1-10 (2010).

[14] Kim, K.I., Kwon, Y.: Single-image super-resolution using sparse 
regression and natural image prior. IEEE Trans. Pattern Anal.Mach. Intell. 32, 1127-1133 (2010).

[15] Yang, J.,Wright, J., Huang, T.,Ma,Y.: Image super-resolution via sparse representation. IEEE Trans. Image Process. 19, 2861-2873 (2010).
[16] Rajaram, S., Gupta, S., Petrovic, N., Huang, S.: Learning-based nonparametric image super-resolution. In: EURASIP Journal on Applied Signal Processing, vol. 2006, Article ID 51306, p. 111doi:10.1155/ASP/2006/5130, (2006). 